Kontext, sondern auch darin, daß Propaganda trotz ihrer Aufwertung unter Kennedy immer noch einen niedrigen Stellenwert im außenpolitischen Instrumentarium insgesamt hat. Die relativ bescheidenen Mittelbewilligungen für sie spiegeln die vergleichsweise höhere Einschätzung des militärischen oder wirtschaftlichen Armes der Außenpolitik in der amerikanischen Gesellschaft. Die Einstellung zur Propaganda als „salesmanship“ und die Verwendung ihrer Techniken, worauf $\mathrm{K}$. zu Recht hinweist, muß oft genug ihre externen Erfolgschancen beeinträchtigen und eben deshalb ihre Wertschätzung in einer Gesellschaft verringern, die meßbaren Erfolg erwartet. - Auch hier bleibt die Analyse etwas vordergründig. Dieser Sachverhalt hätte Überlegungen zum Mangel oder Verlust an Lernfähigkeit der amerikanischen Gesellschaft nahegelegt. Denn wenn auch die Steigerung der Propaganda-Aktivitäten aus einem subjektiven Gefühl der Bedrohung entstanden sein mag, reflektieren diese objektiv doch gerade die (jedenfalls damals noch vorhandene) Mächtigkeit der USA, denn nur der Mächtige kann es sich leisten, nicht $\mathrm{zu}$ lernen und sich nicht $\mathrm{zu}$ adaptieren, diese Last also anderen aufzuzwingen, aber auch das nur bis zu einem Punkt des Bruches oder Umschlages.

So sind insgesamt die eingangs angedeuteten Fragen zu einem so wichtigen und interessanten Gegenstand oft mehr angerissen als durchgeführt. Gerade die theoretische Reflexion bricht verschiedentlich dort $a b$, wo sie interessant zu werden beginnt. Diese Kritik erhebt sich für den, der mit der Materie einigermaßen vertraut ist. Als Einführung in sie und als innerhalb der selbstgesteckten Grenzen ausgezeichnet, materialreich und informativ gearbeitete Studie ist das Buch jedem Interessierten bestens zu empfehlen.

Knud Krakau

\title{
Siegfried Magiera
}

Die Vorwahlen (Primaries) in den Vereinigten Staaten

Demokratisierung von Wahlen und Parteien. Parlamente und Parteien Bd. 3, hrsg. vom Institut für Internationales Recht an der Universität Kiel, Frankfurt 1971, Athenäum Verlag, 174 S. Bibliographie, Index

\section{EBERHARD KÖLSCH}

\section{Vorwahlen}

Zur Kandidatenaufstellung in den USA. Berlin 1972, Duncker und Humblot, Schriften zum Offentlichen Recht, Bd. 187, 166 S. Bibliographie, kein Index

Diese beiden Arbeiten, fast gleichzeitig entstanden, fordern zum Vergleich heraus, da sie denselben Gegenstand behandeln: die amerikanischen Vorwahlen (Vw). Die Vw spielen übrigens für die Präsidentenwahlen, wodurch sie jedem Zeitungsleser auch außerhalb der USA geläufig sind, nur eine untergeordnete und indirekt wirkende Rolle. Obwohl beide Arbeiten von einem juristischen Verlag bzw. Institut in Reihen herausgegeben worden sind, die im allgemeinen juristische Arbeiten (meist Dissertationen) betreuen, stellt sich dieser Vergleich als nicht sehr produktiv heraus, weil es sich um sehr unterschiedliche Ansätze und deshalb praktisch inkommensurable Arbeiten handelt. Das Buch von Kölsch ist im wesentlichen das eines Politikwissenschaftlers, der sich auf moderne Wahlanalysen mittels quantifizierender, statistischer Methoden stützt und sie referierend, interpretierend, die Daten auch neu verarbeitend fortführt. Es ist in erster Linie analytisch, systema- 
tisch, vernachlässigt die historische Dimension weitgehend bis auf gelegentlich kurze Hinweise dort, wo sie nach Ansicht von K. einen Teilaspekt aufzuhellen imstande ist. Die zentrale Fragestellung zielt auf die demokratische Kandidatenaufstellung und demokratische Führungsauslese. Konzentriert, informiert, intelligent, sehr materialreich, eine ausgezeichnete Leistung.

Auch dem Buch von Magiera geht es um den Demokratie-Aspekt bei der Kandidatenaufstellung. Es ist aber analytisch sehr viel weniger scharf. Man könnte es am ehesten als Rechts-deskriptiv bezeichnen. Einfacher zu lesen, weniger befrachtet mit Zahlen und Tabellen, ist es dem Nichtspezialisten gleichwohl durchaus eher zu empfehlen: als Einführung M., danach K. für den Fortgeschrittenen.

M. beschreibt in erster Linie Institutionen, die sie regelnden Rechtsnormen, ihre Wirkungen usw., K. dagegen analysiert ihr Funktionieren im Hinblick auf die zentrale Fragestellung unter Berücksichtigung der wichtigsten Variablen (Bewerber, Parteien, Interessengruppen, Wähler).

M. schildert zunächst die allgemeinen Grundlagen der Vorwahlen (Staatsform und Staatsgewalt, die sehr zahlreichen Wahlämter, die allgemeinen Wahlen und Parteien) und dann in einem besonderen Kapitel ihre geschichtliche Entwicklung, die Demokratisierungsimpulse als Reaktion gegen "machine politics“ des 19. Jahrhunderts, die Entwicklung der relevanten Gesetzgebung im wesentlichen auf Einzelstaatsebene, ergänzt um einige bundesstaatliche Details, sowie die Rechtsprechung. Der Hauptteil behandelt die Erscheinungsformen der Vorwahlen, ein weiterer ihre Auswirkungen; es folgen einige Schlußbemerkungen.

Der Unterschied zwischen beiden Büchern sei an folgendem verdeutlicht: Nach einer Einleitung über die Struktur (Theorie, Funktionen und Organisation) amerikanischer (im Gegensatz zu europäischen) Parteien beschreibt K. im 2. Kapitel auf gerade 12 Seiten die Typen und Varianten von Vw, denen M. den Hauptteil seiner Arbeit widmet. M. definiert kurz als Partei- $\mathrm{V}_{\mathbf{w}}$ „ein der allgemeinen Wahl angeglichenes Verfahren, in dem wahlberechtigte Parteianhänger die Kandidaten der Partei für öffentliche Wahlämter unmittelbar selbst bestimmen“, im Gegensatz zur Aufstellung durch Delegiertenkonvente (65). Es folgen Hinweise auf gesetzliche Regelungen, Rechtsprechung, dann die Beschreibung der Formen der Partei-Vw, closed/open primary, das Registrierungsverfahren bei der ersteren, Einwendungsverfahren, Parteitests, welche Staaten welche Verfahren wählen (und die Vielfalt der Variationen ist in der Tat immer wieder verwirrend), dann noch weitere „Einzelheiten“: Vorschlagsberechtigung, Vorschlagslisten, Gebühren, Vorschläge von einzelnen und von Parteien usw. usw., dann das Verfahren für die nonpartisanVw, die Vw für unabhängige Bewerber, die Konventmethode u. a. m.

K. dagegen - dies alles auf wenigen Seiten vorausgeschickt - fragt im Hinblick auf das Demokratie-Kriterium bei der Kandidatenaufstellung und damit der Rekrutierung politischer Führung nach der Rolle, Funktion und Wirkungsmöglichkeit der Bewerber, der Parteien, Interessengruppen und Wähler. Innerhalb des Kapitels „Bewerber und Vw“ z. B. interessiert ihn besonders der Grad von Wettbewerb unter den Kandidaten, den K. offenbar als Indikator für Demokratie, aber auch Qualität und Effizienz versteht; im einzelnen untersucht K., ob das Vw-Verfahren im Gegensatz zum Konventverfahren wirklich mehr Offenheit verbürgt wie die Vermutung nahelegt; wie wirkt sich das Erfordernis der Unterschriftensammlung und Gebühr aus; einerseits steigert sich der Kandidaten-Wettbewerb mit dem Stimmenanteil einer Partei in "sicheren Wahlkreisen“ (Hochburgen); ist diese 
Tatsache eine kompensatorische Alternative zum fehlenden zwischenparteilichen Wettbewerb in „undemokratischen“ Regionen mit (praktisch) nur einer Partei? Andererseits nehmen auch in Hochburgen nur an etwa der Hälfte der Vw mehr als zwei Bewerber teil. Ein weiterer die Bewerberkonkurrenz determinierender Faktor ist offenbar die Wiederbewerbung von bisherigen Mandataren, sie beeinträchtigt den Wettbewerb; weiter: Welche Rolle spielen in diesem Zusammenhang Bevölkerungsdichte, Grad der Urbanisierung, politische Kultur (auch regionale), wirtschafts-konjunkturelle Entwicklung, Intensität der Vw-Konkurrenz selbst in/ zwischen den Parteien (im Hinblick auf die Hauptwahl), Qualifikation, Wahlkampfkosten und -ausgaben etc.? Zur Rolle der Parteien etwa: Wie wirken die Parteien (später: Interessengruppen), inoffiziell oder institutionalisiert, in der Phase der Rekrutierung von Kandidaten (innerhalb welcher K. fünf Modalitäten unterscheidet) und danach in der Phase der Selektion (unter den rekrutierten Kandidaten bis zur Hauptwahl) auf den Nominierungsproßeß ein? Schmälert/ steigert diese Einwirkung der Parteien/Interessengruppen den intendierten/erreichten Konkurrenz-/Demokratisierungseffekt? - Das alles wird jeweils unter Berücksichtigung der außerordentlichen Vielfalt der Vw-Typen und -regelungen der Einzelstaaten sorgfältig und wo immer möglich mit statistischer Aufarbeitung vorhandenen empirischen Materials untersucht - eine sehr eindrucksvolle Leistung. Insgesamt bejaht $\mathrm{K}$. den Demokratisierungseffekt der Vw, jedenfalls im Vergleich zum Verfahren der Benennung durch Delegierte und unter amerikanischen Bedingungen. Der Frage ihrer Übertragbarkeit auf die Bundesrepublik Deutschland geht er am Schluß sehr behutsam unter Berücksichtigung relevanter struktureller, historisch-politischer, rechtlicher usw. Unterschiede nach.

Knud Krakau

\section{Christian Egbert Weber}

Die Integration eines Kontinents als Problem: Amerika, Europa.

Berlin 1971, Duncker \& Humblot, 94 S.

Wirtschaft und Gesellschaft in den Vereinigten Staaten von Amerika. Berlin 1961, Duncker \& Humblot, 255 S.

Es handelt sich hier um zwei eigenwillige aber in ihrer Art sehr anregende Bücher. Der Verfasser, der viele Jahre in Amerika gelebt hat und ein guter Kenner der gesellschaftlichen und kulturellen Probleme und Entwicklungen der USA ist, versagt sich gängigen Einordnungen. Er distanziert sich sehr bewußt von neueren wissenschafts-theoretischen Positionen und inhaltlichen Umgewichtungen in der Analyse gesellschaftlich-historischer Entwicklungen in den USA, die er aber mit der polemisch-verkürzenden Formel "Spezialistentum und Positivismus" doch wohl etwas unzureichend charakterisiert. Wenn schon solche Kategorien, dann würde auf W's eigene Position am besten die des konservativen Konsensus-Theoretikers passen (vgl. die "einheitliche Denkungsart“ S. 30 (1971) oder die Bezugnahme auf L. Hartz' „Liberal Tradition“ S. 33 ib., wiederholte Hinweise auf die starke Integrationskraft der amerikanischen Kultur, z. B. 43 ff. ib. usw.).

In dem insgesamt interessanteren (aktuelleren und konzentrierteren) Bande 1971 unternimmt W. einen Vergleich zwischen den USA und Europa, dessen amerikanische Seite im Bande 1961 in zahlreichen Einzelaspekten breiter ausgeführt ist, man 